\title{
R.F. PLANAR MAGNETRON SPUTTERED ZnO FILMS II: ELECTRICAL PROPERTIES
}

F. R. BLOM*, F. C. M. VAN DE POL $\dagger$, G. BAUHUIS AND TH. J. A. POPMA

University of Twente, Department of Electrical Engineering, P.O. Box 217, 7500 AE Enschede (The Netherlands)

(Received October 23, 1990; accepted February 12, 1991)

The electrical properties of r.f. planar magnetron sputtered $\mathrm{ZnO}$ films are studied by means of current-voltage, capacitance-voltage and Van der Pauw measurements.

These films are applied as piezoelectric transducers in micromechanical sensors and actuators. Their piezoelectric behaviour strongly depends on the electric properties.

A conduction model for the polycrystalline $\mathrm{ZnO}$ layers is presented. This model gives a good description of the electrical behaviour, and is useful in understanding the piezoelectric properties of the films studied.

\section{INTRODUCTION}

Within our group, $\mathrm{ZnO}$ films are applied as piezoelectric transducers in micromechanical sensors and actuators. The films are sputtered on substrates of silicon, silicon oxide (crystalline and amorphous), metal (aluminium, silver and gold) or quartz, from a zinc target in a pure oxygen atmosphere with a planar r.f. magnetron sputter unit.

It was concluded that the piezoelectric behaviour of these $\mathrm{ZnO}$ films was much worse than had been expected ${ }^{1-3}$. Suggested possible causes were (i) lower piezoelectric coefficients for the film than the bulk values, (ii) losses due to direct currents and (iii) structural deviations.

We studied the structural, electrical and piezoelectric properties of the films and their relations to the deposition parameters. Deposition and structural properties are discussed in Part I ${ }^{4}$. A study on the piezoelectric behaviour has been reported elsewhere ${ }^{5}$.

In this paper (Part II) we deal with electric properties. In Section 2, a model for the electrical conduction of the films is presented. In Section 3, experiments concerning current-voltage, capacitance-voltage and Van der Pauw measurements

* Present address: Oce Nederland B. V. Venlo, The Netherlands.

† Present address: Schlumberger Industries, SMR, B. P. 620-05, 92542 Montrouge, France. 
are described. Results are given and discussed in Section 4, while conclusions are presented in Section 5.

\section{CONDUCTION MODEL}

Sputtered $\mathrm{ZnO}$ films are polycrystalline, the individual crystals growing preferentially with their crystallographic $c$-axis perpendicular to the substrate. $\mathrm{ZnO}$ belongs to the hexagonal wurzite crystal type, having $6 \mathrm{~mm}$ symmetry. It is generally treated as an n-type semiconductor, as a result of excessive zinc, with interstitial zinc atoms and/or oxygen vacancies occupied by electron pairs, serving as donor states. ${ }^{6}$

\subsection{Basic models from the literature}

Several conduction models for polycrystalline semiconductors can be found in literature. Basic models are proposed by Volger ${ }^{7}$ and Petritz ${ }^{8}$. Reviews of these models, their limitations and refinements, are given by Kazmerski ${ }^{9}$ and by Orton and Powell ${ }^{10}$.

In the Volger model, the polycrystalline material is divided into identical cubes (the grains) consisting of two different materials: the bulk material at the centre of the cube (the kernel), and the boundary material which surrounds this centre (grain boundary region) (Fig. 1(a)). Generally, the grain boundaries of a polycrystalline semiconductor contain surface states, due to defects or absorbed ions, which can trap free carriers from the bulk material. This results in band bending near the grain boundary (see Fig. l(b)) and depletion of the boundary material. Thus the boundary region can be described electrically in terms of two back-to-back Schottky barriers.
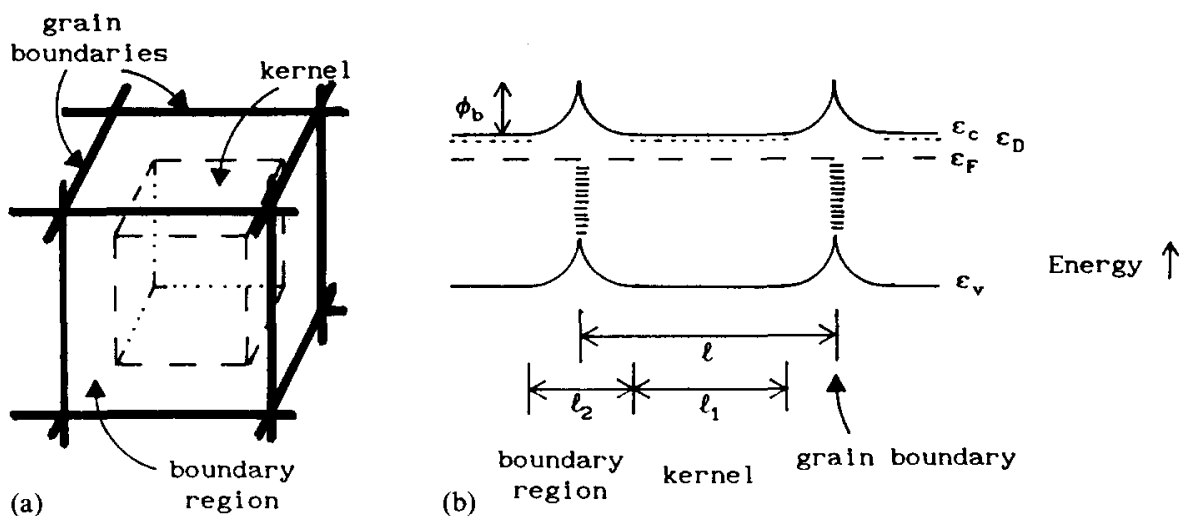

Fig. 1. (a) Geometric model of a polycrystalline semiconductor, showing the cubic grains comprising a kernel and a grain boundary region. (b) Energy band representation of an n-type polycrystalline semiconductor (grain size $l$, grain barrier height $\phi_{\mathrm{b}}$ ).

If the mechanism for conduction across the barriers is known, then expressions for the effective resistivity of the polycrystalline material can be derived. Basic assumptions thereby are that both the mean free path $\lambda$ of the carriers and the Debye length $L_{\mathrm{D}}$ are small compared with the grain size. 


\subsection{Geometric model of the $\mathrm{ZnO}$ films}

It was shown in Part $\mathrm{I}^{4}$ that $\mathrm{ZnO}$ films grown on amorphous $\mathrm{SiO}_{2}$ substrates consist of broad columnar grains with their long axis perpendicular to the substrate, containing hardly any boundaries parallel to the substrate. Therefore we can simplify the three-dimensional Volger model with cubes to a two-dimensional model with columns (Fig. 2). These columns, with (grain) width $l$ and height $d_{\mathrm{z}}$ (film thickness), comprise semiconducting kernels (width $l_{1}$ ), surrounded by permanently depleted boundary regions (width $l_{2}$ ).

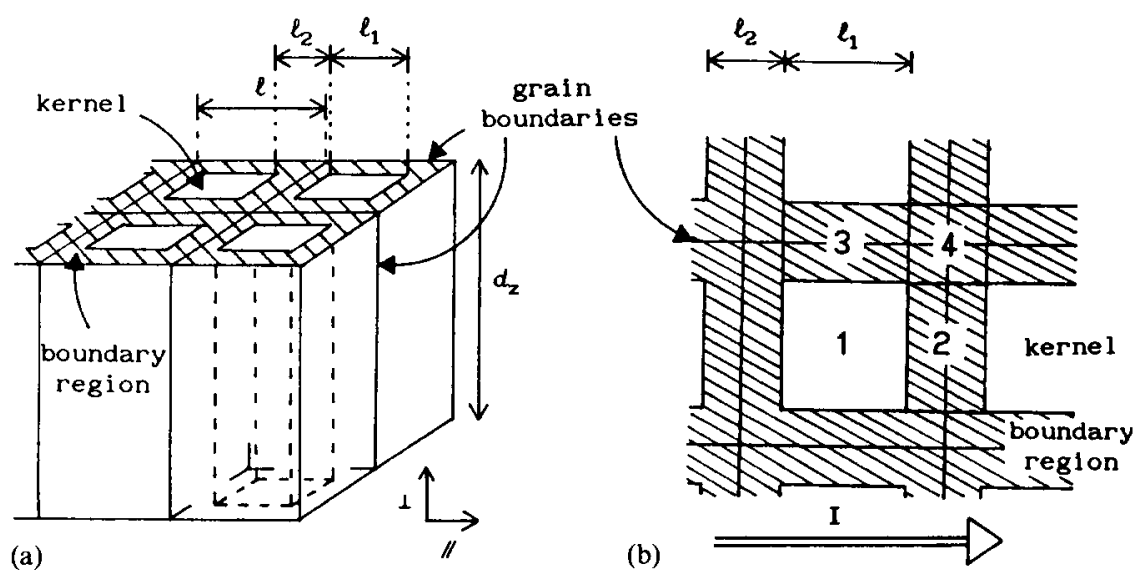

Fig. 2. (a) Geometric model of a $\mathrm{ZnO}$ layer showing the columnar grains comprising a kernel and a grain boundary region. (b) Top view showing the different regions.

\subsection{Free carrier concentration and trapping state density}

A basic assumption is that all donor states are ionized, and thus the free carrier concentration $n_{\mathrm{z}}$ inside the kernel equals the donor concentration $N_{D}$. Considering the energy differences between the donor levels and the conduction band, typically $0.05 \mathrm{eV}$, this appears to be a valid assumption ${ }^{6}$. A relation between the density $n_{\mathrm{t}}$ of trapping states at the grain boundaries and $n_{\mathrm{z}}$ can be derived by equating the number of donor states in the boundary region with the captured electrons in the trapping states at the boundaries. We then find

$$
n_{\mathrm{t}}=\frac{1}{2}\left\{\frac{(\gamma+1)^{2}-1}{(\gamma+1)^{2}}\right\} n_{\mathrm{z}} l
$$

with

$$
\gamma=l_{2} / l_{1} \text { and } l=l_{1}+l_{2}
$$

\subsection{Resistivity parallel and perpendicular to the substrate}

The resistivity $\rho_{1}$ of the kernel is much smaller than that $\rho_{2}$ in the grain boundary region. Using the geometric model of Fig. 2, we find the following for the effective resistivity $\rho_{\|}$parallel to the substrate:

$$
\rho_{\|}=\frac{(\gamma+1) \rho_{1 \|}+\gamma(\gamma+1) \rho_{2 \|}}{\gamma \rho_{1} / \rho_{2 \|}+\gamma^{2}+\gamma+1}
$$


Also, for the resistivity $\rho_{\perp}$ perpendicular to the substrate,

$$
\rho_{\perp}=(\gamma+1)^{2} \rho_{1 \perp} \frac{\rho_{2 \perp}}{\left\{(\gamma+1)^{2}-1\right\} \rho_{1 \perp}+\rho_{2 \perp}}
$$

where $\rho_{1 \|}$ and $\rho_{1 \perp}$ are the resistivities of the kernel parallel and perpendicular respectively to the substrate. Generally, these two differ for the two crystal directions. The same holds for $\rho_{2_{\|}}$and $\rho_{2,}$, the resistivities of the grain boundary region parallel and perpendicular to the substrate.

\subsection{Conduction mechanism across the barriers}

The dominant conduction mechanism across a barrier is determined by the tunnel constant $E_{00}$ (ref. 11):

$$
E_{00}=(e \hbar / 2)\left(N_{\mathrm{D}} / \varepsilon m^{*}\right)^{1 / 2}
$$

with $m^{*}$ the carrier effective mass, $\varepsilon=\varepsilon_{\mathrm{r}} \varepsilon_{0}$ the permittivity of the semiconductor $\left(\varepsilon_{\mathrm{r}}\right.$ is the relative permittivity and $\varepsilon_{0}$ the permittivity of vacuum), $e$ the electron charge and $\hbar=h / 2 \pi$ ( $h$ is the Planck constant).

If $E_{00} \ll k T$, with $k$ the Boltzmann constant and $T$ the absolute temperature, then thermionic emission is dominant; only free carriers with an energy exceeding the height $\phi_{\mathrm{b}}$ of the barrier contribute to the conduction. At room temperature this is the case for $N_{\mathrm{D}}<10^{24} \mathrm{~m}^{-3}$. As will be seen, thermionic emission is the relevant conduction mechanism in our case.

For larger values of $E_{00}$, tunnelling through the barrier becomes more important. We can then distinguish thermionic field emission and field emission. Field emission only plays a role at very high doping levels $\left(N_{\mathrm{D}}>10^{28} \mathrm{~m}^{-3}\right)$ or low temperatures.

\subsection{Effective resistivity}

The current $i$ flowing over a barrier when a voltage $V_{\mathrm{b}}$ is applied is given by

$$
i=i_{0}\left\{\exp \left(\frac{e V_{\mathrm{b}}}{k T}\right)-1\right\}
$$

where for thermionic emission $i_{0}$ is given by ${ }^{10}$

$$
i_{0}=A A^{\prime} \frac{n_{\mathrm{z}}}{N_{\mathrm{c}}} \exp \left(-\frac{\phi_{\mathrm{b}}}{k T}\right)
$$

with $A$ the area of the surface perpendicular to the current, $\phi_{\mathrm{b}}$ the barrier height, $A^{\prime}$ the Richardson constant and $N_{\mathrm{c}}$ the effective density of states in the conduction band. $A^{\prime}$ and $N_{\mathrm{c}}$ are given by

$$
A^{\prime}=4 \pi m^{*} e(k T)^{2} / h^{3}
$$

and

$$
N_{\mathrm{c}}=2\left(2 \pi m^{*} k T / h^{2}\right)^{3 / 2}
$$

For a small applied voltage $\left(V_{\mathrm{b}} \ll k T / e\right)$, the voltage drop across the back-to-back double barrier is equally divided between both barriers. In this case the differential 
conductance $g_{2}$ across the barrier is given by

$$
g_{2}=\frac{\partial i}{\partial V_{\mathrm{b}}}=i_{0} \frac{e}{2 k T}=\frac{l_{1} d_{\mathrm{z}} e A^{\prime} n_{\mathrm{z}}}{2 k T N_{\mathrm{c}}} \exp \left(-\frac{\phi_{\mathrm{b}}}{k T}\right)
$$

With $1 / g_{2}=\rho_{2} l_{2} / l_{1} d_{\mathrm{z}}$ and using eqns. (2), (3), (8) and (9), we can find a relation between $\rho_{\|}$and $\phi_{\mathrm{b}}$ :

$$
\rho_{\|}=\frac{1}{\gamma^{2}+\gamma+1} \rho_{1 \|}+\frac{\gamma}{\gamma^{2}+\gamma+1} \frac{\gamma+1}{\gamma} \frac{\left(8 \pi m^{*} k T\right)^{1 / 2}}{l e^{2} n_{\mathrm{z}}} \exp \left(\frac{\phi_{\mathrm{b}}}{k T}\right)
$$

\section{EXPERIMENTS}

All $\mathrm{ZnO}$ films are sputtered under s-conditions ${ }^{4}$ :

substrate temperature $\quad 400-450^{\circ} \mathrm{C}$

r.f. forward sputter power $1800 \mathrm{~W}$

oxygen pressure $\quad 0.86 \mathrm{~Pa}$

target-substrate distance $45 \mathrm{~mm}$

\subsection{Current-voltage I-V measurements}

Current-voltage $I-V$ measurements are performed, using an HP Parameter Analyser, in order to determine the resistivity $\rho_{\perp}$ perpendicular to the substrate. The $\mathrm{ZnO}$ is sandwiched between two aluminium electrodes, as shown in Fig. 3(a). The sample temperature is varied using a heater-controller.

\subsection{Capacitance-voltage $C$-V measurements}

Standard capacitance-voltage $C-V$ measurements ${ }^{12-14}$ are performed with an HP 4194A impedance-gain phase analyser at a spot frequency of $1 \mathrm{MHz}$, using a metal-oxide-semiconductor (MOS) structure with $\mathrm{ZnO}$ as the semiconductor (see Fig. 3(b)). A sample comprises a $4 \mu \mathrm{m}$ thick layer of $\mathrm{ZnO}$ on top of a chemically vapour deposited $\mathrm{SiO}_{2}$ layer, sandwiched between two aluminium electrodes.

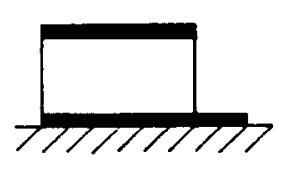

(a)

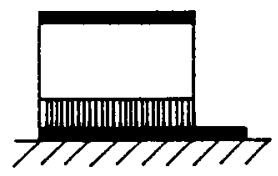

(b)

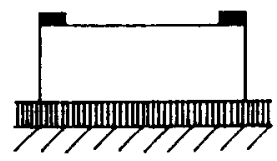

(c)

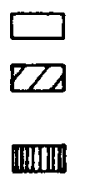

Aluminium
ZnO, 4 um thick

Substrate:

thermal $\mathrm{SiO}_{2}$

CVD $\mathrm{SiO}_{2}$

Fig. 3. Sample structures for (a) $I-V$ measurement, (b) $C-V$ measurement and (c) Van der Pauw measurement.

Figure 4 shows an equivalent electrical circuit representing this MOS structure. $C_{\mathrm{d}}$ corresponds to the permanently depleted grain boundary regions, $C_{\mathrm{z}}$ to the induced depletion layer in the kernels. $R_{\mathrm{z}}$ to the semiconducting kernels, and $C_{\mathrm{ox}}$ to the $\mathrm{CVD} \mathrm{SiO}$ layer.

The thickness $W_{\mathrm{d}}$ of the induced depletion layer varies with the applied d.c. voltage, and so does $C_{\mathbf{z}} \cdot W_{\mathrm{d}}$ reaches its maximum $W_{\max }$ when an inversion layer is 


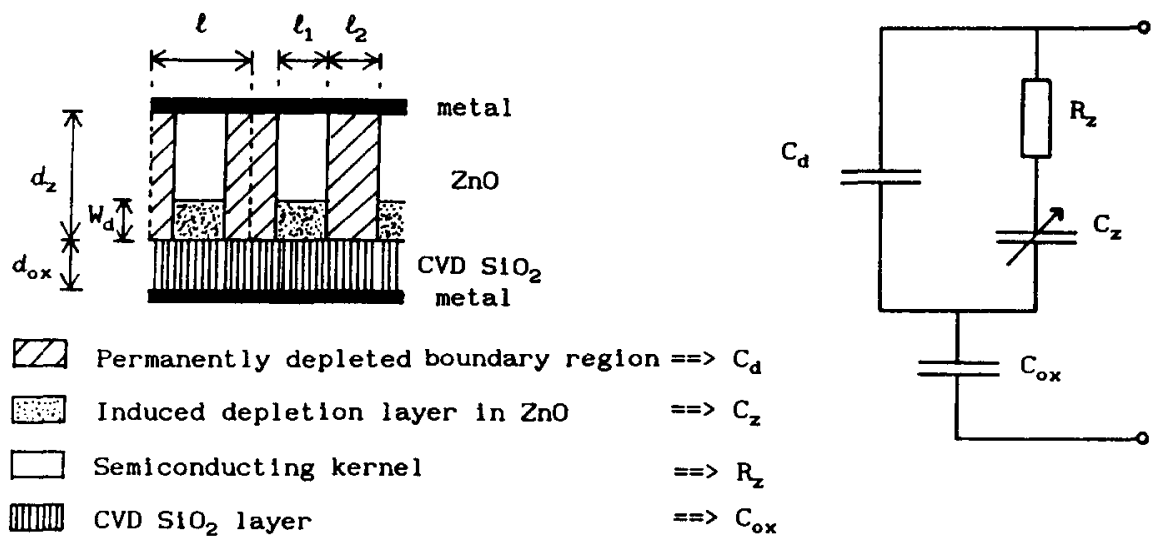

Fig. 4. Cross-section of the MOS structure and the equivalent electrical circuit.

created at the $\mathrm{ZnO}-\mathrm{SiO}_{2}$ interface. $W_{\max }$ is a function of $n_{z}{ }^{14,15}$ :

$$
W_{\max }=\left\{\frac{4 \varepsilon k T}{e^{2} n_{\mathrm{z}}} \ln \left(\frac{n_{\mathrm{z}}}{n_{\mathrm{i}}}\right)\right\}^{1 / 2}
$$

with $n_{i}$ the intrinsic free carrier concentration.

Fixed or mobile charges may be present in the $\mathrm{SiO}_{2}$ or at the $\mathrm{ZnO}-\mathrm{SiO}_{2}$ interface, causing a shift in the flat band voltage $V_{\mathrm{FB}}$ (the difference between the work functions of metal and semiconductor) and deforming the $C-V$ curve (Fig. 5).

A normalized capacitance $C_{\mathrm{n}}$ is defined as the ratio of the total capacitance $C_{\text {MOs }}$ of the MOS structure to the capacitance $C_{\text {ox }}$ of the oxide layer. This $C_{\mathrm{n}}$ reaches a minimum value $C_{\mathrm{n}-\min }$ when $W_{\mathrm{d}}$ reaches $W_{\max }$. For $C_{\mathrm{n}-\min }$ we find

$$
\begin{aligned}
C_{\mathrm{n}-\min } & =\frac{C_{\mathrm{Mos}}\left(W_{\mathrm{d}}=W_{\max }\right)}{C_{\mathrm{ox}}} \\
& =\frac{C_{\mathrm{d}}+C_{\mathrm{z}}}{C_{\mathrm{d}}+C_{\mathrm{z}}+C_{\mathrm{ox}}} \\
& =\left\{1+\frac{\varepsilon_{\mathrm{ox}}}{\varepsilon_{\mathrm{z}}} \frac{W_{\max }}{d_{\mathrm{ox}}} \frac{(\gamma+1)^{2}}{\left\{(\gamma+1)^{2}-1\right\} W_{\max } / d_{\mathrm{z}}+1}\right\}^{-1}
\end{aligned}
$$

with $\varepsilon_{\mathrm{ox}}$ and $\varepsilon_{\mathrm{z}}$ the permittivities of the $\mathrm{SiO}_{2}$ and $\mathrm{ZnO}$ respectively and $d_{\mathrm{ox}}$ the thickness of the chemically vapour deposited $\mathrm{SiO}_{2}$ layer. $C_{\mathrm{n}-\min }$ is derived from the $C-V$ plot, and depends on the parameters $\gamma$ and $n_{z}$, since $W_{\max }=W_{\max }\left(n_{z}\right)$.

\subsection{Van der Pauw measurements}

In order to determine the resistivity $\rho_{\|}$parallel to the substrate, the Van der Pauw method is used ${ }^{16}$. The samples (see Fig. 3(c)) comprise squares of $4 \mu \mathrm{m}$ thick $\mathrm{ZnO}$, grown on chemically vapour deposited $\mathrm{SiO}_{2}$. Aluminium point electrodes are deposited on top of these squares, at the corners.

The measurements are performed inside a dark and grounded box. The sample temperature is varied using a heater-controller. 


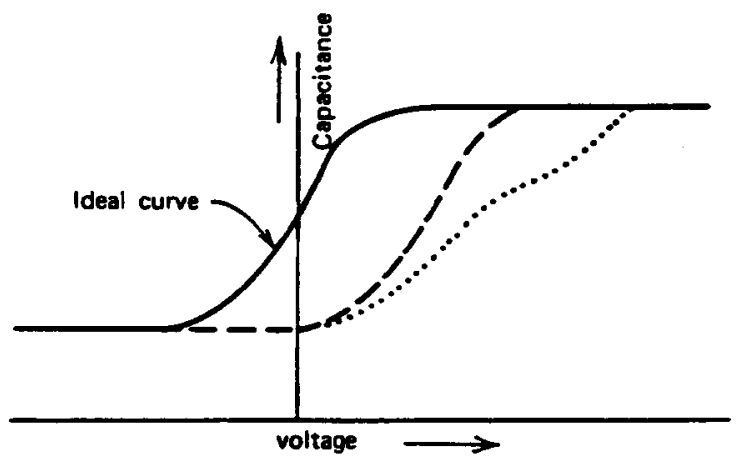

Fig. 5. $C-V$ curves, ideal (—), and distorted as a result of a fixed charge (_- - $(-)$ and as a result of a mobile charge $(\ldots \ldots)^{15}$.

\subsection{Determination of $\mathrm{n}_{z}, \mathrm{n}_{\mathrm{t}}, \gamma$ and $\rho_{1 \perp}$}

Using the $I-V, C-V$ and Van der Pauw measurements, $\rho_{\perp}, C_{\mathrm{n}-\min }$ and $\phi_{\mathrm{b}}$ are determined. The grain width $l$ is measured by scanning electron microscopy $(\mathrm{SEM})^{4}$. In order to determine $n_{z}, n_{1}, \gamma$ and $\rho_{1 \perp}$, four equations are required. We have derived three of them: eqns. (1), (4) and (13). The fourth equation is the Poisson formula for a single barrier, relating $\phi_{\mathrm{b}}, n_{\mathrm{z}}, l$ and $\gamma^{14,15}$ :

$$
\phi_{\mathrm{b}}=\frac{e^{2} n_{\mathrm{z}} l^{2}}{8 \varepsilon_{\mathrm{z}}}\left(\frac{\gamma+1}{\gamma}\right)^{2}
$$

Using an iterative method, the four (non-linear) equations are solved for $n_{z}, n_{t}, \gamma$ and $\rho_{1 \perp}$. Values for the constants $\varepsilon_{\mathbf{z}}, \varepsilon_{\mathrm{ox}}, n_{\mathrm{i}}$ and $m^{*}$ are obtained from the literature.

\section{RESULTS AND DISCUSSION}

\subsection{I-V measurements}

All $I-V$ measurements reveal an ohmic behaviour of the $\mathrm{Al}-\mathrm{ZnO}-\mathrm{Al}$ structure, indicating ohmic contacts between $\mathrm{ZnO}$ and aluminium.

Figure 6 shows a plot of $\rho_{\perp} v s$. temperature $T$, in which $\rho_{\perp}$ appears to be independent of the temperature. This is in agreement with our assumption that no grain boundaries are present parallel to the substrate.

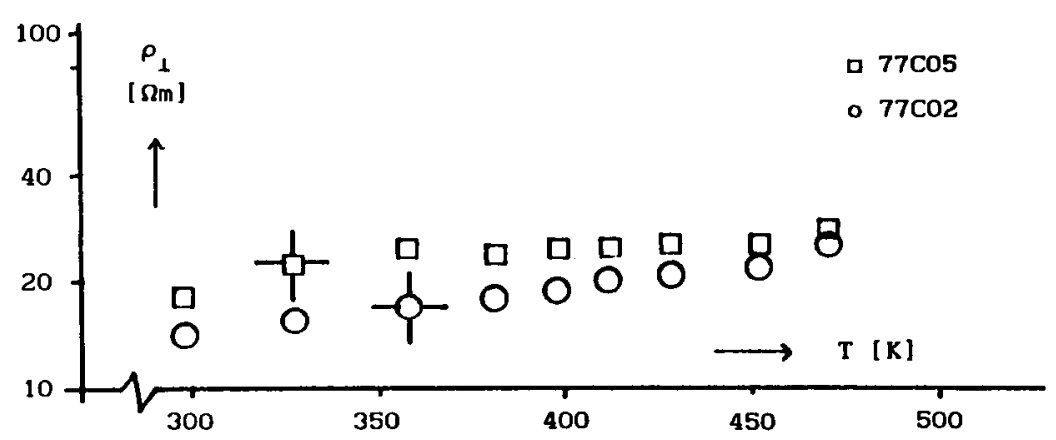

Fig. 6. Resistivity perpendicular to the substrate $v s$. temperature for two $\mathrm{Al}-\mathrm{ZnO}-\mathrm{Al}$ structures. 


\section{2. $C-V$ measurements}

$C-V$ plots were recorded for a number of samples with different surface areas. A typical plot is shown in Fig. 7. A shift $\Delta V_{\mathbf{F B}}$ in the flat band voltage from its ideal position is observed. We measured an average shift of $5 \pm 1 \mathrm{~V}$, which means we are dealing with negative charge carriers trapped in the $\mathrm{SiO}_{2}$ or at the $\mathrm{ZnO}-\mathrm{SiO}_{2}$ interface.

The average value of $C_{\mathrm{n}-\min }$, as derived from these $C-V$ plots, equals $0.5 \pm 0.1$.

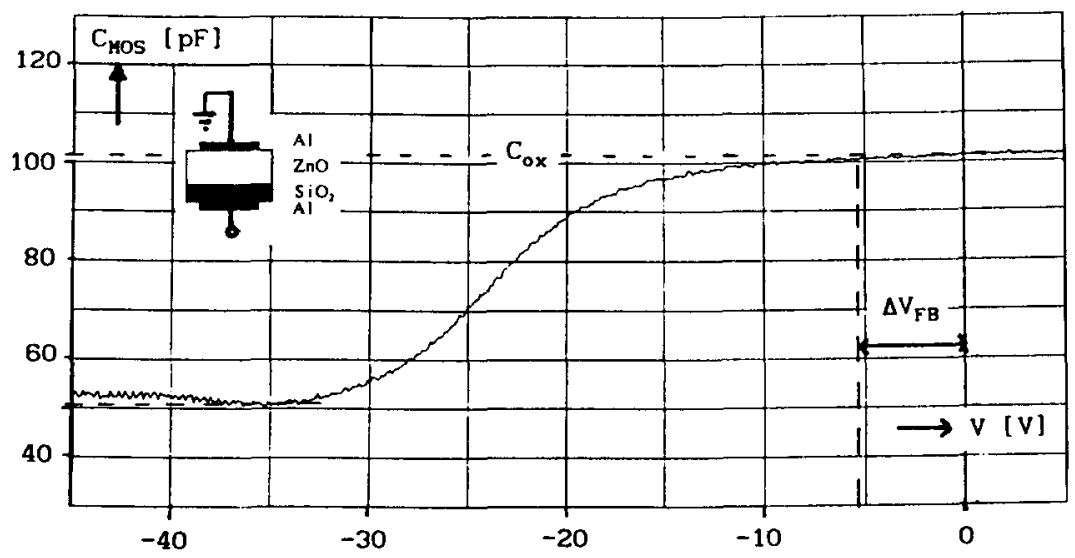

Fig. 7. Typical $C-V$ plot.

\subsection{Van der Pauw measurements}

For a number of samples with different surface areas, $\rho_{\|} v s$. temperature was measured. As expected, $\rho_{\|}$decreases with temperature, whereby we can conclude that the resistivity parallel to the substrate is determined by the barrier resistivity.

It appears that the term $\rho_{\|} /\left(\gamma^{2}+\gamma+1\right)$ in eqn. (11) can be neglected compared with $\rho_{\|}$for temperatures below $400 \mathrm{~K}$. Therefore, $\phi_{\mathrm{b}}$ can be derived from the slope of a plot of $\ln \left(\rho_{\|} / T^{1 / 2}\right)$ vs. 1/T. In Fig. 8, plots of $\ln \left(\rho_{\|} / T^{1 / 2}\right) v s$. 1/T are shown for a number of measurements performed on the same sample within a short period of time. From measurement to measurement, both $\rho_{\|}$and $\phi_{\mathrm{b}}$ decrease, but seem to reach equilibrium values at the last measurements. In Table I, measured values of $\rho_{\|}$ at room temperature and calculated values of $\phi_{\mathrm{b}}$ are listed.

We can conclude that $\rho_{\|}(T)$ and $\phi_{\mathrm{b}}$ are strongly dependent on the thermal history of the sample. Shapira et al. ${ }^{17}$ and Tansley and coworkers ${ }^{11,18-20}$ described the processes which cause these phenomena. The major adsorbed species at the grain boundaries is carbon, which becomes chemisorbed as $\mathrm{CO}_{2}$ in the presence of oxygen. This $\mathrm{CO}_{2}$ is ionized to $\mathrm{CO}_{2}{ }^{-}$by trapping a free electron. By means of illumination, this ion-molecule can be neutralized and molecular $\mathrm{CO}_{2}$ desorbs from the sample. Thus, the number of trapping states diminishes and the barrier height and consequently the resistivity decrease. When the illumination is removed, oxygen again adsorbs on unattached carbon sites, creating new trapping states, and the barrier height will again increase.

Tansley et al. ${ }^{20}$ reported that these sorption-trapping-desorption processes 


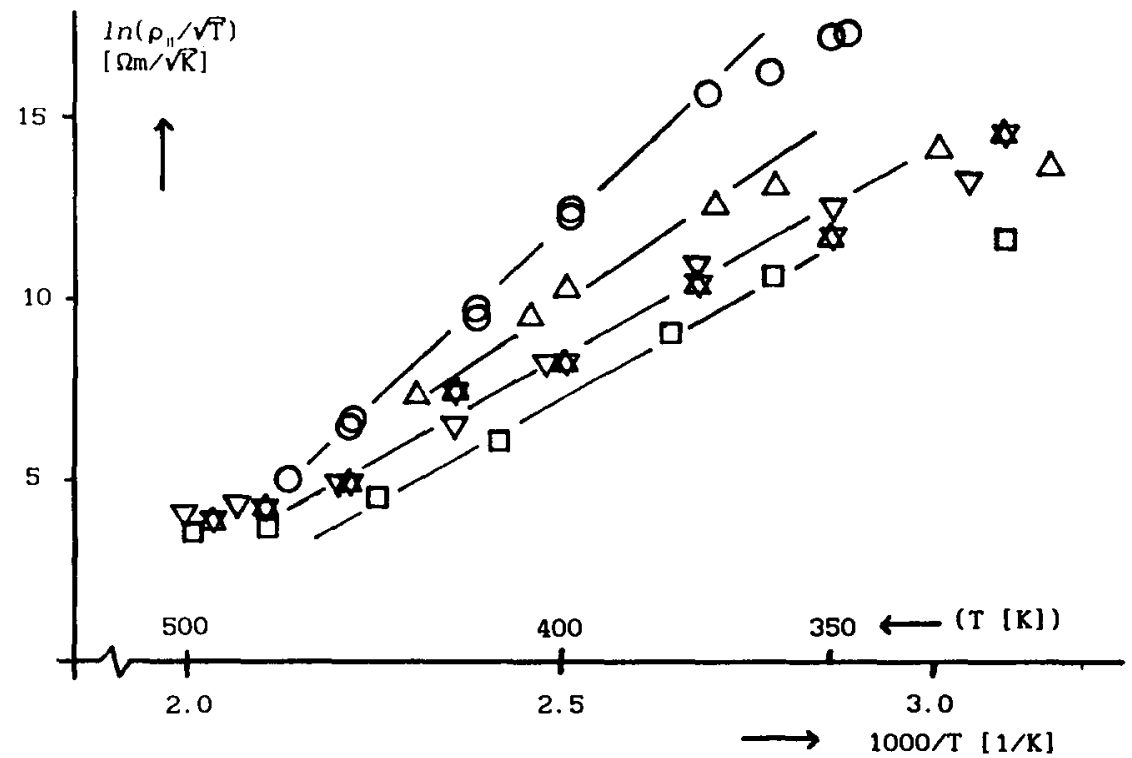

Fig. 8. $\ln \left(\rho_{\|} / T^{1 / 2}\right)$ vs. $1 / T$ for the same sample (77P11), measured several times in succession: symbols as in Table I.

TABLE I

DATA FOR MEASUREMENTS OF $\rho_{\|}$FOR SAMPLE 77P1 I

\begin{tabular}{llll}
\hline $\begin{array}{l}\text { Number symbol } \\
\text { in Fig.8 }\end{array}$ & Date of measurement & $\begin{array}{l}\phi_{\mathrm{b}} \\
(\mathrm{eV})\end{array}$ & $\begin{array}{l}\rho_{\|} \text {at room temperature } \\
(\Omega \mathrm{m}) \pm 50 \%\end{array}$ \\
\hline 10 & June 24 & $1.7 \pm 0.1$ & $>10^{10}$ \\
$2 \triangle$ & June 28 & $1.2 \pm 0.2$ & $>10^{9}$ \\
$3 \square$ & June 29 & $0.9 \pm 0.1$ & $10^{8}$ \\
$4 \nabla$ & June 30 & $0.9 \pm 0.1$ & $10^{8}$ \\
$5 \square$ & July 3 & $0.9 \pm 0.2$ & $10^{7}$ \\
\hline
\end{tabular}

$\pm 50 \%$.

also occur when the sample is heated instead of illuminated. They found that the conductivity exhibited a strong increase with temperature above $500 \mathrm{~K}$, in agreement with our observations (Fig. 8). They deduced values for the barrier height ranging from an initial value of $0.85 \mathrm{eV}$ to $0.6 \mathrm{eV}$ after illumination or heating ${ }^{18}$. We found values of $1.7 \mathrm{eV}$ and $0.9 \mathrm{eV}$ respectively (Table I).

\subsection{Calculation of $\mathrm{n}_{z}, n_{t}, \gamma$ and $\rho_{1 \perp}$} and $\rho_{1 \perp}$.

The values of $l, \rho_{\perp}, C_{\mathrm{n}-\min }$ and $\phi_{\mathrm{b}}$ being known, we can now calculate $n_{\mathbf{z}}, n_{\mathfrak{t}}, \gamma$

For $m^{*}$ we take $0.27 m_{\mathrm{e}}$, with $m_{\mathrm{e}}$ the electron rest mass ${ }^{6}$. Using eqn. (9), we find $N_{\mathrm{c}}=3.5 \times 10^{24} \mathrm{~m}^{-3}$.

Using the well-known formula for $n_{\mathrm{i}}{ }^{14}$,

$$
n_{\mathrm{i}}=\left(N_{\mathrm{c}} N_{\mathrm{v}}\right)^{1 / 2} \exp \left(-\varepsilon_{\mathrm{g}} / 2 k T\right)
$$


with $N_{\mathrm{c}}=N_{\mathrm{v}}, N_{\mathrm{v}}$ the effective density of states in the valence band and $\varepsilon_{\mathrm{g}}=3.2 \mathrm{eV}$, the gap energy of $\mathrm{ZnO}$, we find $n_{\mathrm{i}}=6 \times 10^{-4} \mathrm{~m}^{-3}$.

For the permittivities $\varepsilon_{\mathrm{ox}}$ and $\varepsilon_{\mathrm{z}}$, we have $3.5 \varepsilon_{0}$ and $11.3 \varepsilon_{0}$ respectively ${ }^{21}$, with $\varepsilon_{\mathrm{z}}$ the permittivity at constant stress.

The values are summarized as follows:

$$
\begin{aligned}
l & =0.3 \pm 0.1 \mu \mathrm{m} & & \text { (SEM observations } \left.^{4}\right) \\
\rho_{\perp} & =20 \pm 5 \Omega \mathrm{m} & & (I-V \text { measurements }) \\
C_{\mathrm{n}-\min } & =0.5 \pm 0.1 & & (C-V \text { measurements }) \\
\phi_{\mathrm{b}} & =1.2 \pm 0.3 \mathrm{ev} & & \text { (Van der Pauw measurements) }
\end{aligned}
$$

With the iterative method mentioned previously, we find

$$
\begin{aligned}
n_{\mathrm{z}} & =5 \times 10^{23} \mathrm{~m}^{-3}( \pm 50 \%) \\
n_{\mathrm{t}} & =5 \times 10^{16} \mathrm{~m}^{-2}( \pm 50 \%) \\
\gamma & =1.0 \pm 0.2 \\
\rho_{1 \perp} & =5 \Omega \mathrm{m}( \pm 40 \%) .
\end{aligned}
$$

The calculated values for $n_{\mathrm{z}}$ and $n_{\mathrm{t}}$ correspond well to values found by other researchers for sputtered $\mathrm{ZnO}$ films ${ }^{18,20,22}$.

\subsection{Further results}

Figure 9 shows a combined plot of $\rho_{1 \perp}$ and $\rho_{\|} v s$. temperature. At higher temperatures, $\rho_{\|}$reaches the same order of magnitude as $\rho_{1 \perp}$. We can conclude that, at higher temperatures, the barriers play a less important role and the effective resistivity is determined by the bulk resistivity $\rho_{1 \|}$.

The mobility $\mu_{\perp}$ of the free carriers inside the kernel can be determined from the measured value of $\rho_{1 \perp}$ and the calculated value of $n_{z}$. It follows from $1 / \rho_{1 \perp}=n_{z} e \mu_{1 \perp}$ that $\mu_{1 \perp}=(3 \pm 1) \times 10^{-6} \mathrm{~m}^{2} \quad \mathrm{~V}^{-1} \mathrm{~s}^{-1}$. This is a lower value than reported by other researchers for ("bulk") polycrystalline $\mathrm{ZnO}$ (e.g. $\mu=1 \times 10^{-4} \mathrm{~m}^{2} \mathrm{~V}^{-1} \mathrm{~s}^{-1}$ (ref. 6)), but in good agreement with the values Horsthuis $^{22}$ found for sputtered films.

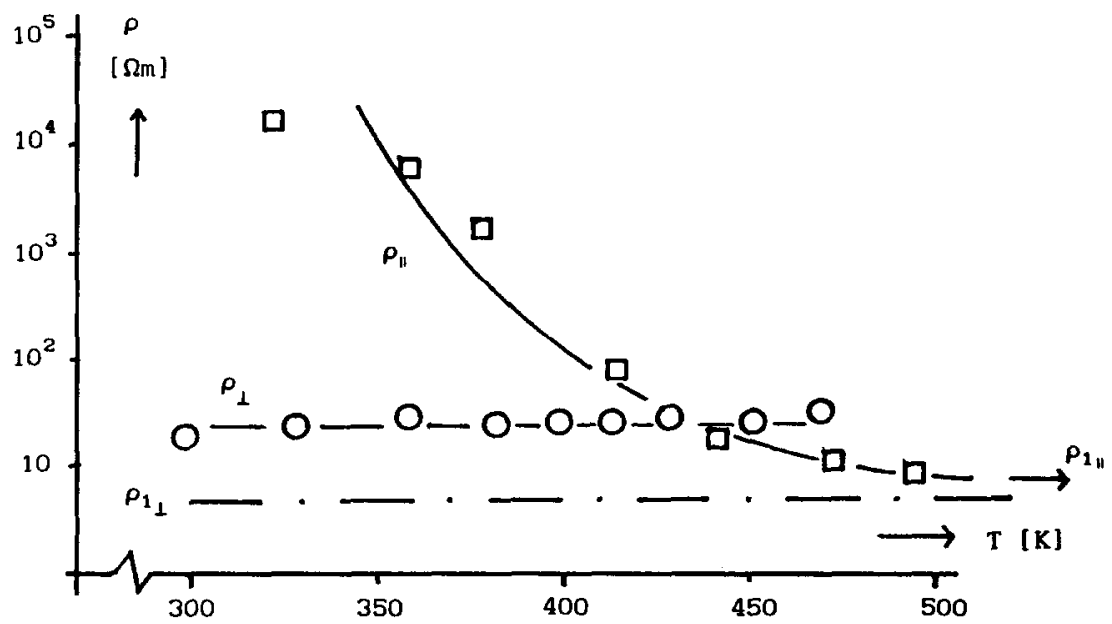

Fig. 9. Resistivity, perpendicular and parallel to the substrate, for a $\mathrm{ZnO}$ layer (wafer 77). 
$W_{\max }$, the maximum thickness of the depletion layer, as calculated from eqn. (15), equals $0.08 \mu \mathrm{m}( \pm 50 \%)$. The calculated width $l_{2}$ of the boundary regions (see Fig. 2) equals $0.15 \mu \mathrm{m}( \pm 30 \%)$. We can conclude that $l_{2}$ has reached its maximum value $2 W_{\max }$.

\subsection{Verifying assumptions}

The electrical parameters of the film being known, the basic assumptions can be verified.

From ${ }^{14} \lambda=\mu m^{*} c / e$, with $c$ the velocity of light in vacuum and $\mu$ the mobility of the electrons, taking for $\mu$ the derived value of $\mu_{1 \perp}=3 \times 10^{-6} \mathrm{~m}^{2} \mathrm{~V}^{-1} \mathrm{~s}^{-1}$, we find that the mean free path $\lambda$ equals $2 \mathrm{~nm}$. (Since $\rho_{1 \|} \approx \rho_{1 \perp}, \mu_{1 \|}$ will be of the same order of magnitude as $\mu_{1 \perp}$ ). The Debye length can be calculated using ${ }^{14,15}$ $L_{\mathrm{D}}=\left(\varepsilon_{\mathrm{z}} k T / e^{2} n_{\mathrm{z}}\right)^{1 / 2}$. We find $L_{\mathrm{D}}=5 \mathrm{~nm}$.

The grain size $l$ equals $0.3 \mu \mathrm{m}$ for $\mathrm{ZnO}$ grown on amorphous substrates and a few hundredths of a micron for $\mathrm{ZnO}$ grown on crystalline substrates ${ }^{4}$. It can be concluded that both $\lambda$ and $L_{\mathrm{D}}$ are small (two orders of magnitude less) compared with the grain size, as assumed.

The tunnel constant $E_{00}$, as calculated from eqn. (2), equals $0.009 \mathrm{eV}$, being somewhat smaller than $k T$ (about $0.025 \mathrm{eV}$ at room temperature). Nevertheless, the contribution of thermionic field emission is still negligible. We can conclude that thermionic emission is the dominant conduction mechanism across the grain barriers, as assumed.

The calculated value of $\gamma$ equals $1.0 \pm 0.2$, which means that $l_{1} \approx l_{2}$, implying that the grains are not completely depleted, and the kernels occupy some $25 \%$ of the cross-sectional area of a grain.

We conclude that all assumptions are valid.

\section{CONCLUSTONS}

In this paper we have proposed a conduction model for polycrystalline $\mathrm{ZnO}$ films based on models for polycrystalline semiconductors known from the literature ${ }^{7-10}$. The films consist of columnar grains with a semiconducting kernel and a completely depleted boundary region, and hardly any grain boundaries parallel to the substrate. The conduction across a boundary is described in terms of a Schottky barrier with thermionic emission as the dominant conduction mechanism.

In order to verify this model, current-voltage, capacitance-voltage and Van der Pauw measurements were performed on $\mathrm{ZnO}$ films grown on chemically vapour deposited $\mathrm{SiO}_{2}$ and on aluminium. With the aid of the model, in combination with grain size measurements ${ }^{4}$, we found the following for the electrical parameters:

$\begin{array}{ll}\text { free electron concentration } & n_{\mathrm{z}}=5 \times 10^{23} \mathrm{~m}^{-3}( \pm 50 \%) \\ \text { density of trapping states } & n_{\mathrm{t}}=5 \times 10^{16} \mathrm{~m}^{-2}( \pm 50 \%) \\ \text { grain barrier height } & \phi_{\mathrm{b}}=1.2 \pm 0.2 \mathrm{eV} \\ \text { depletion ratio } & \gamma=1.0 \pm 0.2 \\ \text { resistivity in the kernel } & \rho_{1 \perp}=5 \Omega \mathrm{m}( \pm 40 \%) \\ \text { electron mobility in the kernel } & \mu_{1 \perp}=(3 \pm 1) \times 10^{-6} \mathrm{~m}^{2} \mathrm{~V}^{-1} \mathrm{~s}^{-1}\end{array}$


The resistivity parallel to the substrate appears to be strongly dependent on the thermal history of the sample. The heating of the sample causes adsorbed $\mathrm{CO}_{2}{ }^{-}$at the grain boundaries to neutralize and desorb. Thus the density of trapping states is diminished and the barrier height, as well as the resistivity, decreases. After cooling down, $\mathrm{O}_{2}$ adsorbs at carbon sites, restoring the original electrical properties.

We conclude that the proposed model gives a good description of the electric behaviour of the polycrystalline $\mathrm{ZnO}$ films studied.

\section{ACKNOWLEDGMENTS}

The authors would like to thank H. Postma for helping with the Van der Pauw measurements and $J$. Baxter for his help in preparing the manuscript of this paper. This research was performed within the program of the Dutch Foundation for Fundamental Research on Matter (FOM) and was partly sponsored by the Netherlands Technology Foundation (STW).

\section{REFERENCES}

1 J. G. Smits, H. A. C. Tilmans, K. Hoen, H. Mulder, J. van Vuuren and G. Boom, Sens. Actuators, 4 (1983) 565.

2 S. Bouwstra, University of Twenet, personal communication (1986).

3 F. R. Blom, Resonant silicon beam force sensor, Ph.D. Thesis, University of Twente, 1989.

4 F. C. M. van de Pol, F. R. Blom and Th. J. A. Popma, Thin Solid Films, 202 (1991).

5 F. R. Blom, D. J. Yntema, F. C. M. van de Pol, M. Elwenspoek, J. H. J. Fluitman and Th. J. A. Popma, Sens. Actuators A, 21-23(1989) 226.

6 W. Hirschwald, in E. Kaldis (ed.), Current Topics in Materials Science, Vol. 7, North-Holland, Amsterdam, 1981, pp. 143482.

7 J. Volger, Phys. Rev., 79 (1950) 1023.

8 R. L. Petritz, Phys. Rev., 104 (1956) 1508.

9 L. L. Kazmerski, Polycrystalline and Amorphous Thin Films and Devices, Academic Press, New York, 1980.

10 J. W. Orton and M. J. Powell, Rep. Prog. Phys., 43 (1980) 1263.

11 T. L. Tansley, D. F. Neeley and C. P. Foley, Thin Solid Films, 117 (1984) 19.

12 K. H. Zaininger and F. P. Heiman, Solid State Technol., 13 (1970) 49.

13 K. H. Zaininger and F. P. Heiman, Solid State Technol., 13 (1970) 46.

14 S. M. Sze, Semiconductor Devices; Physics and Technology, Wiley, New York, 1985.

15 R. S. Muller and T. I. Kamins, Device Electronics for Integrated Circuits, Wiley, New York, 2nd edn., 1986.

16 L. J. Van der Pauw, Philips Res. Rep., 13 (1958) 1.

17 Y. Shapira, S. M. Cox and D. Lichtman, Surf. Sci., 54 (1976) 43.

18 T. L. Tansley and D. F. Neeley, Thin Solid Films, 121 (1984) 95.

19 T. L. Tansley and S. J. T. Owen, J. Appl. Phys., 55 (1984) 454.

20 T. L. Tansley, C. P. Foley and D. F. Neeley, Thin Solid Films, 121 (1984) L85.

21 Landolt-Börnstein Numerical Data and Functional Relationships in Science and Technology, Vol. III-11, Springer, Berlin, 1979.

22 W. H. G. Horsthuis, Investigations on periodically perturbed optical waveguides, Ph.D. Thesis. University of Twenet, 1987. 